\title{
Cooperative Connectivity Models for Wireless Relay Networks
}

\author{
John Boyer, Member, IEEE, David D. Falconer, Fellow, IEEE, and Halim Yanikomeroglu, Member, IEEE
}

\begin{abstract}
This paper considers the ways that cooperating terminals can be connected to each other in wireless relay networks and the constraints imposed by the availability of different system resources. A framework is developed that exposes the relationship between constraints on available system resources and the achievable combinations of communication links between cooperating terminals. Cooperative connectivity models defined by the achievable combinations of links are derived, associated with their minimum cost constraint sets, and mapped to diversity techniques presented in the literature. The constraints considered are the available number of orthogonal relaying channels, the ability of terminals to diversity combine signals on a single common channel, the ability of terminals to diversity combine signals on orthogonal channels, the ability of terminals to transmit signals on multiple orthogonal channels, and the ability of terminals to cancel the effects of interhop interference.
\end{abstract}

Index Terms - Cooperative connectivity, cooperative diversity, cooperative relaying, multihop relaying, spatial diversity.

\section{INTRODUCTION}

$\mathbf{R}$ ECENT findings in the literature have shown that the performance of wireless relay networks can be improved through the application of distributed spatial diversity techniques that leverage cooperation between wireless terminals. Multi-user diversity [3] and virtual antenna arrays [15] achieve spatial diversity by relaying the signal along multiple routes in parallel. Multihop diversity [8], [9] achieves spatial diversity from the reception of signals that have been transmitted by multiple relays in serial along a single multihop route. Cooperative diversity [39], [40], [42], [61] achieves spatial diversity by sharing information between the source terminal and relay terminals such that each user of the group sends information to the destination using all of the terminals. Coded cooperative diversity [30], [31], [41], [45], [64], [76] uses various coding techniques, including space-time coding [1], to improve the performance over that of basic repetition coding. Various techniques based on the artificial generation of a rich multipath fading environment [19], [23], [48], [55], [59], [62], [63], [68] can be leveraged to reclaim some of the spectral efficiency loss caused by repetition coding over orthogonal relaying channels. Information theoretic results for the classical relay channel [14] have been extended to include more general

Manuscript received September 25, 2005; revised August 8, 2006 and December 11, 2006; accepted January 8, 2007. The associate editor coordinating the review of this letter and approving it for publication was W. Liao. This work has been performed in part in the framework of the IST project IST4-027756 WINNER II, which is partly funded by the European Union. This work was funded by the Natural Sciences and Engineering Research Council (NSERC) of Canada.

The authors are with Carleton University, Department of Systems and Computer Engineering, 1125 Colonel By Drive, Ottawa Ontario, K1S-5B6 Canada (e-mail: jboyer@sce.carleton.ca; ddf@sce.carleton.ca; halim@sce.carleton.ca).

Digital Object Identifier 10.1109/TWC.2007.05742. multi-hop relay channels [21], [38]. Results characterizing the diversity-multiplexing tradeoff of multiple-antenna [77] and multiple-access [65] channels have been extended to halfduplex cooperative channels [4]. Basic strategies for cooperation between wireless terminals have been extended to include cascaded [22], [28], [58], multihop multi-branch [57], and general [10] cooperation strategies. These distributed spatial diversity techniques contrast with non-distributed techniques such as classical MIMO transmission that require individual terminals to host multiple antennas.

Each of the distributed spatial diversity techniques proposed in the literature places different requirements on the wireless terminal hardware capabilities, channel availability, and multiple access schemes used to implement mesh connectivity between terminals, and thus places different requirements on the system resources that must be available. Therefore, system resource constraints that limit the ways that terminals can be connected to each other also constrain the distributed spatial diversity techniques that can be applied. Additionally, the cooperation between terminals needed to support each technique can often be achieved with different combinations of system resources. Instead of generating results for one specific assumed set of available system resources, this paper explicitly specifies the system resources that must be available for terminals to be connected to each other in different ways, and therefore to utilize different distributed spatial diversity techniques.

This paper develops a framework for modeling the ways that terminals can be connected to each other in wireless relay networks and the relationship between constraints on the available system resources and achievable cooperative connectivity. The cooperative connectivity of a wireless relay network is defined as the set of communication links between pairs of terminals that are used in the transmission of an information signal from a source terminal to a destination terminal. Relay terminals are defined to cooperate with the source terminal when they transmit a signal that helps the destination terminal to successfully decode the original information signal. The system resource constraints considered in this paper are the available number of orthogonal relaying channels, the ability of relays and destinations to diversity combine incident signals on a single common channel, the ability of relays and destinations to diversity combine incident signals on different orthogonal channels, the ability of transmitters to transmit signals on multiple orthogonal channels, and the ability of receivers to cancel the effects of interhop interference [9]. Generally, it is of significant relevance to understand the different ways that cooperating terminals can be connected to each other in wireless relay networks, which of those options for cooperative connectivity show the most promise for further investigation 
and eventual adoption, and which system resources can enable those options for cooperative connectivity in the most efficient manner. The developed framework is a valuable first step in the analysis of these issues, and enables the formulation of interesting and relevant, but previous unexpressed, questions with respect to cooperative connectivity.

For example, the framework allows us to formulate and solve the following classes of problems: 1) given an available set of system resources, determine the achievable cooperative connectivity, 2) given a desired level of cooperative connectivity, determine the possible (and lowest cost) sets of system resources that can be used to achieve it, 3) given a baseline set of system resources and level of cooperative connectivity, determine the impact on the achievable cooperative connectivity of incrementally adding or removing different system resources, and 4) given a baseline set of system resources and level of cooperative connectivity, determine the possible (and lowest cost) sets of system resources that can be incrementally added or removed to achieve different levels of cooperative connectivity. The first problem class and its inverse, the second problem class, consider the core relationship between system resources and achievable cooperative connectivity. The third problem class and its inverse, the fourth problem class, consider the value of reusing existing capabilities of the network infrastructure as incremental modifications are made. Each of the different system resources has an associated cost, so it is important that existing investment is leveraged when modifications are made to network infrastructure. We note that these problem classes do not inherently express the philosophy that more connectivity is better, but are simply concerned with the relationship between achievable cooperative connectivity and available system resources. Even so, in the context of a given source-destination terminal pair when the system resources used are constant, it is clear that more cooperative diversity will result in better performance. However, this is not necessarily the case when considered in the context of a system with many source-destination terminal pairs, where many other factors such as spatial reuse and inter-user interference must be considered.

Although the focus of this paper is on uncoded systems, the developed framework is equally applicable to systems that employ traditional source and channel coding techniques. These coding techniques can be employed regardless of the ways that cooperating terminals can be connected to each other. However, the choice of coding technique should depend on the composite characteristics of the multihop relay channel formed by a given set of cooperating terminals and communication links between them. For cooperative coding techniques, such as those presented in [30], [31], [41], [45], [64], [76], where different relays generate different re-encoded versions of the original transmitted information signal, there are strong requirements on the ways that cooperating terminals can be connected to each other. For example, the distributed spacetime code protocol of [41] requires that all relays be directly connected to both the source and destination in parallel. General analysis of the cooperative connectivity requirements for different cooperative coding techniques is outside the scope of this paper. However, the framework can be used to determine the possible sets of system resources that can be used to achieve the required cooperative connectivity of a given technique.

The developed framework does not address the relationship between cooperative connectivity and spatial reuse of channels, a mechanism that can be applied in wireless relay networks to improve the overall spectral efficiency. Spatial reuse of channels occurs when different signals can be transmitted concurrently on the same channels in spatially separated portions of multihop paths (or networks). However, spatial reuse is less feasible in systems where terminals are connected by long direct communication links that do not allow spatial partitioning of the network, especially when using a multiple access scheme that does not allow spatial overlap of concurrent transmissions on the same channel. Systems with higher levels of cooperative connectivity, which generally have more long direct communication links and enjoy higher levels of spatial diversity, are generally less able to leverage spatial reuse due to a corresponding larger region of interference. This tradeoff between improved spatial diversity and improved spatial reuse is an important topic for further analysis.

The organization of the remainder of the paper is as follows. Section 2 describes the considered system resource constraints in detail. Section 3 analyzes the possible combinations of system resource constraints and derives cooperative connectivity models defined by the achievable combinations of communication links between cooperating wireless terminals. Section 4 derives the sets of constraints that result in the different cooperative connectivity models while minimizing the system cost (minimum cost constraint sets) and presents cooperative connectivity model transition diagrams following these minimum cost constraint sets. Section 5 describes the mapping of the cooperative connectivity models to various distributed spatial diversity techniques presented in the literature in order to highlight the general richness of the problem domain. Section 6 provides some concluding remarks.

\section{System Resource Constraints}

The considered system resource constraints are described in detail in this section. Options for each constraint are introduced, along with their corresponding relative system cost and connectivity impact. In all cases, constraint options with lower cost have higher connectivity impact. Connectivity impact is defined as the reduction in achievable cooperative connectivity caused by absence of a system resource, and is measured in comparison to a fully connected (complete) relay network with links between all terminals. The term 'preceding terminal' denotes any terminal that is earlier along the multihop transmission path than the candidate terminal. The term 'following terminal' denotes any terminal that is later along the multihop transmission path than the candidate terminal.

\section{A. Number of Channels Available}

This constraint defines the number of orthogonal relaying channels available for each source-destination pair. The halfduplex nature of wireless terminal hardware requires that each relay transmit and receive with different channels, implying a minimum of two orthogonal channels. When the modulation 
scheme, total power, and rate are kept constant, use of more than two orthogonal channels for relaying increases the system cost since more bandwidth is necessary to achieve a given rate of transmission for each source-destination pair. However, when the symbol rate loss due to subdivision of the original bandwidth can be compensated for by an improved end-toend SNR, the requirement for additional orthogonal channels may not necessarily result in an increase in overall required bandwidth.

- $N$ Channels Available (NCA): The source and relays transmit using $N$ orthogonal channels, where $N+1$ is the number of terminals. There is no connectivity impact.

- K Channels Available (KCA): The source and all relays transmit using $K$ orthogonal channels, where $2<K<$ $N$. The connectivity impact is that each terminal may only be connected to preceding terminals that transmit on a different subset of channels ( $K-1$ possible channels for relays and $K$ possible channels for destinations).

- 2 Channels Available (2CA): The source and all relays transmit using 2 orthogonal channels. The connectivity impact is that each terminal may only be connected to preceding terminals that transmit on a different subset of channels ( 1 possible channel for relays and 2 possible channels for destinations).

\section{B. Common Channel Combination}

This constraint defines the ability of terminals to diversity combine incident signals from multiple preceding terminals on a single common channel. Common channel combination can be achieved using various techniques including space-time coding, random relay phase rotation, and artificial multi-path generation with adaptive equalization, spatial processing, or RAKE reception [19], [23], [48], [55], [59], [62], [63], [68]. One interesting result of many of these references is that diversity combination can be achieved even without the use of orthogonal channels, although in many cases, depending on the specific channel conditions, there may be some degradation of performance in comparison to that of maximal ratio combining. Use of common channel combination increases the system cost since more advanced common channel processing and combination hardware is required at cooperating terminals.

- Relay Common Channel Combination (RCC): Relays are able to perform common channel combination. There is no connectivity impact.

- No Relay Common Channel Combination (NRCC): Relays are not able to perform common channel combination. The connectivity impact is that each relay may only be connected to one preceding terminal on each channel.

- Destination Common Channel Combination (DCC): Destinations are able to perform common channel combination. There is no connectivity impact.

- No Destination Common Channel Combination (NDCC): Destinations are not able to perform common channel combination. The connectivity impact is that each destination may only be connected to one preceding terminal on each channel.

\section{Orthogonal Channel Combination}

This constraint defines the ability of terminals to diversity combine incident signals from multiple preceding terminals on different orthogonal channels. Orthogonal channel combination can be achieved using classical combination techniques and possibly buffering of multiple orthogonal channels. Use of orthogonal channel combination increases the system cost since classical combination hardware is required at cooperating terminals.

- Relay Orthogonal Channel Combination (ROC): Relays are able to perform orthogonal channel combination. There is no connectivity impact.

- No Relay Orthogonal Channel Combination (NROC): Relays are not able to perform orthogonal channel combination. The connectivity impact is that each relay may only be connected to the subset of preceding terminals that transmit on one common channel.

- Destination Orthogonal Channel Combination (DOC): Destinations are able to perform orthogonal channel combination. There is no connectivity impact.

- No Destination Orthogonal Channel Combination (NDOC): Destinations are not able to perform orthogonal channel combination. The connectivity impact is that each destination may only be connected to the subset of preceding terminals that transmit on one common channel.

\section{Multiple Channel Transmission}

This constraint defines the ability of terminals to transmit a given signal on multiple orthogonal channels. Use of multiple channel transmission increases the system cost since more complex channel transmission hardware may be required at cooperating terminals (for example, transmitting concurrently at multiple frequencies) and each terminal that transmits on multiple channels generates additional energy and interference (when the modulation scheme and rate are kept constant).

- Multiple Channel Transmission (MCT): Terminals are able to transmit on multiple orthogonal channels. There is no connectivity impact.

- No Multiple Channel Transmission (NMCT): Terminals are not able to transmit on multiple orthogonal channels. The connectivity impact is that each terminal may only be connected to the subset of following terminals that receive on one common channel.

\section{E. Interhop Interference Cancellation}

This constraint defines the ability of receivers to cancel the effects of interhop interference created by the retransmission of signals on the same channel at different hops along a multihop transmission path. Interhop interference is a special case of intersymbol interference (ISI) that affects wireless relay networks where channels are reused [9], and therefore in theory it is expected that practical mitigation techniques will be based on those used for ISI, including traditional equalization techniques. Use of interhop interference cancellation increases the system cost since more complex equalization (mitigation) hardware is required at cooperating terminals. 


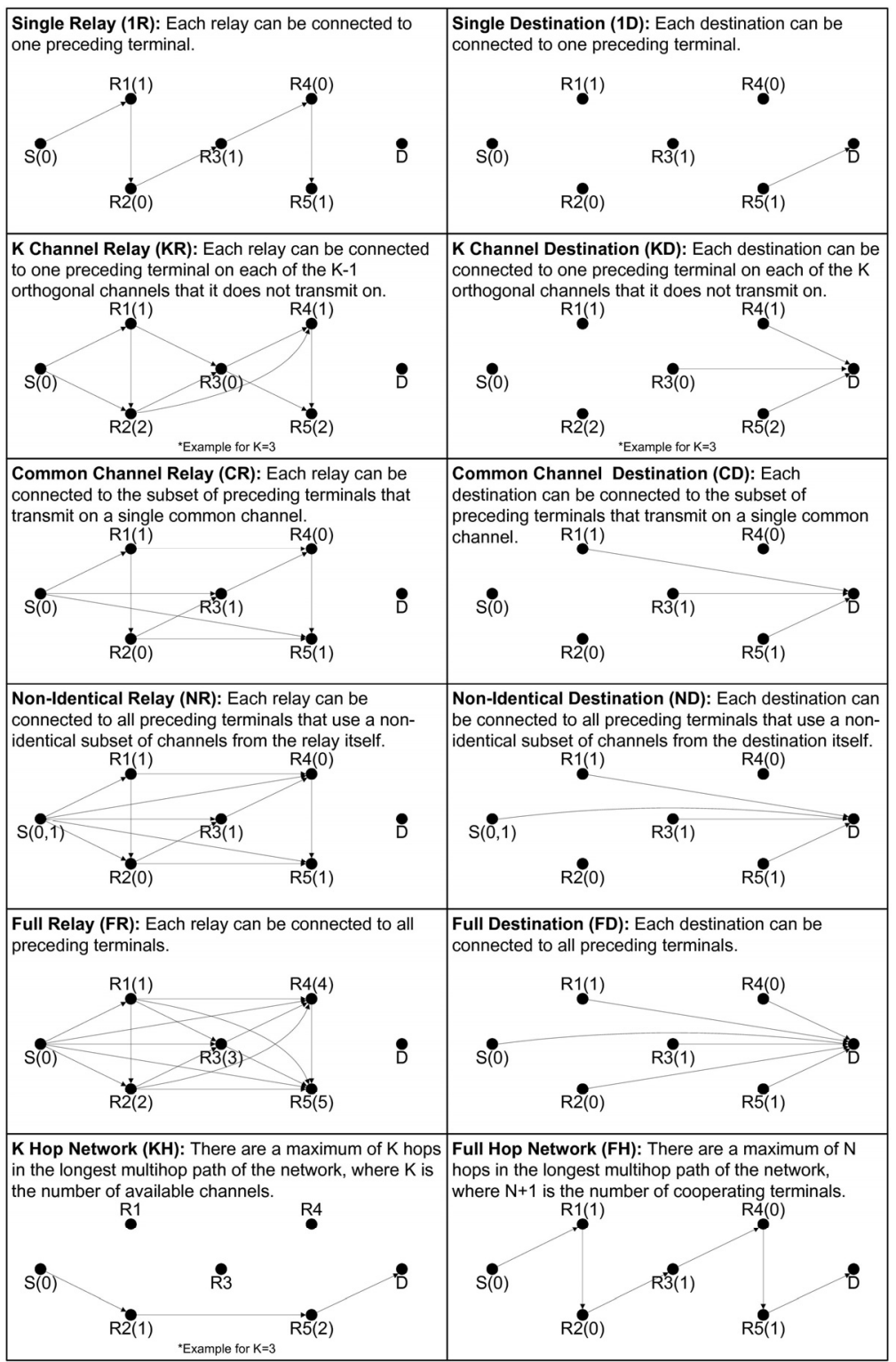

Fig. 1. Cooperative connectivity model terminology examples.

Note that the analysis of this constraint is somewhat simplistic. First, it does not consider that in practice channel reuse may be possible without interhop interference cancellation in some circumstances due to sufficient spatial separation or attenuation between terminals. Second, it is assumed that interference cancellation is perfect when channels are reused at different hops within a single multihop path. Although this is not feasible in practice, even partial interference cancellation allows the same level of cooperative connectivity to be achieved, therefore resulting in the same cooperative connectivity models.

- Interhop Interference Cancellation (IIC): Terminals are able to cancel interhop interference. There is no connectivity impact.

- No Interhop Interference Cancellation (NIIC): Terminals are not able to cancel interhop interference. The connectivity impact is that networks with $K$ channels available have a maximum of $K$ hops in the longest multihop path of the network, since it is not possible to reuse channels within a single multihop path.

\section{Cooperative Connectivity Models}

A set of resultant cooperative connectivity models can be derived from the possible system resource constraint combinations. The cooperative connectivity models are fully characterized according to three parameters: the achievable connectivity of the relays, the achievable connectivity of the destination, and the maximum achievable length of the longest multihop path of the network. The cooperative connectivity models can therefore be identified using the form ' $x \mathrm{R} y \mathrm{D} z \mathrm{H}$ ', where ' $x \mathrm{R}$ ' indicates the achievable connectivity of the relays, ' $y \mathrm{D}$ ' indicates the achievable connectivity of the destination, and ' $z \mathrm{H}$ ' indicates the maximum achievable length of the longest multihop path of the network. Fig. 1 describes the possible values of $x, y$, and $z$ used when characterizing the achievable cooperative connectivity of the models and 


\begin{tabular}{|c|c|c|}
\hline $\begin{array}{c}\text { \# Channels } \\
\text { Available }\end{array}$ & $\begin{array}{c}\text { Cooperative Connectivity } \\
\text { Equations }\end{array}$ & $\begin{array}{l}\text { Minimum Cost Constraint Set } \\
\text { Equations }\end{array}$ \\
\hline $\begin{array}{l}\text { K Channels } \\
\text { Available }\end{array}$ & $\begin{aligned} x \mathrm{R}: & \text { if (NRCC \& NROC) then } x=1 \\
& \text { else if (NRCC\& ROC) then } x=\mathrm{K} \\
& \text { else if (RCC\& NROC\& NMCT) then } x=\mathrm{C} \\
& \text { else if (RCC\& (ROC | MCT)) then } x=\mathrm{N} \\
y \mathrm{D}: & \text { if (NDCC \& NDOC) then } y=1 \\
& \text { else if (NDCC\& DOC) then } y=\mathrm{K} \\
& \text { else if (DCC\& NDOC\& NMCT) then } y=\mathrm{C} \\
& \text { else if (DCC\& (DOC | MCT)) then } y=\mathrm{F} \\
z \mathrm{H}: & \text { if (IIC) then } z=\mathrm{F} \\
& \text { else if (NIIC) then } z=\mathrm{K}\end{aligned}$ & 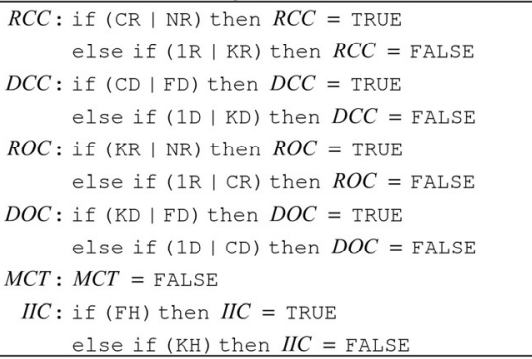 \\
\hline $\begin{array}{l}2 \text { Channel } \\
\text { Available }\end{array}$ & $\begin{aligned} x \mathrm{R}: & \text { if (NRCC I NIIC) then } x=1 \\
& \text { else if (RCC \& NMCT\&IIC) then } x=\mathrm{C} \\
& \text { else if (RCC\& MCT \& IIC) then } x=\mathrm{N} \\
y \mathrm{D}: & \text { if (NDCC\& NDOC) then } y=1 \\
& \text { else if (NDCC\& DOC) then } y=2 \\
& \text { else if (DCC\& NDOC\& NMCT) then } y=\mathrm{C} \\
& \text { else if (DCC\& NDOC\& MCT) then } y=\mathrm{N} \\
& \text { else if (DCC \& DOC) then } y=\mathrm{F} \\
z \mathrm{H}: & \text { if (IIC) then } z=\mathrm{F} \\
& \text { clsc if (NIIC) then } z=2\end{aligned}$ & $\begin{aligned} R C C: & \text { if (CR | NR) then } R C C=\text { TRUE } \\
& \text { else if (1R) then } R C C=\text { FALSE } \\
D C C: & \text { if }(\mathrm{CD}|\mathrm{ND}| \mathrm{FD}) \text { then } D C C=\text { TRUE } \\
& \text { else if }(1 \mathrm{D} \mid \text { 2D) then } D C C=\text { FALSE } \\
R O C: & R O C=\text { FALSE } \\
D O C: & \text { if }(2 \mathrm{D} \mid \mathrm{FD}) \text { then } D O C=\text { TRUE } \\
& \text { else if (ID | CD | ND) then } D O C=\text { FALSE } \\
M C T: & \text { if (NR | ND) then } M C T=\text { TRUE } \\
& \text { else } M C T=\text { FALSE } \\
I C C: & \text { if (FH) then IIC = TRUE } \\
& \text { else if (2H) then } I C=\text { FALSE }\end{aligned}$ \\
\hline $\begin{array}{l}\text { N Channels } \\
\text { Available }\end{array}$ & $\begin{array}{l}x \mathrm{R}: \quad \text { if }(\mathrm{ROC} \mid(\mathrm{RCC} \& \mathrm{MCT})) \text { then } x=\mathrm{F} \\
\quad \text { else } x=1 \\
y \mathrm{D}: \quad \text { if }(\mathrm{DOC} \mid(\mathrm{DCC} \& \mathrm{MCT})) \text { then } y=\mathrm{F} \\
\quad \text { else } y=1 \\
z \mathrm{H}: \quad z=\mathrm{F}\end{array}$ & $\begin{aligned} R C C: & R C C=\text { FALSE } \\
D C C: & D C C=\text { FALSE } \\
R O C: & \text { if }(F R) \text { then } R O C=\text { TRUE } \\
& \text { else if }(1 \mathrm{R}) \text { then } R O C=\text { FALSE } \\
D O C: & \text { if (FD) then DOC = TRUE } \\
& \text { else if (1D) then DOC = FALSE } \\
M C T: & M C T=\text { FALSE } \\
I C C: & I I C=\text { FALSE }\end{aligned}$ \\
\hline
\end{tabular}

Fig. 2. Cooperative connectivity and minimum cost constraint set equations.

shows graphical examples of the cooperative connectivity terminology, with transmitting channel allocations indicated in brackets. Note that other channel allocations are possible that achieve the same connectivity. The relationship between this parametrization and the maximum achievable diversity order of the network is not simple, although higher levels of achievable relay and especially achievable destination connectivity definitely do correspond to an increase in maximum achievable diversity order. It is shown in [12] that the maximum achievable diversity order of wireless relay networks is constrained by either the terminal or cut set with the minimum number of incident inter-terminal links, depending on the class of relaying method.

The cooperative connectivity equations presented in Fig. 2 specify the relationship between the system resource constraints and the characterizing parameters of the cooperative connectivity models. These equations address the first problem class listed in the introduction: given an available set of system resources, determine the achievable cooperative connectivity. The complete set of achievable models is shown in Figs. 35 , and discussed in more detail in the next section. The number of available channels (NCA, KCA, and 2CA) has a structuring influence on the connectivity impact of the other constraints. The results are therefore presented classified by the number of available channels in order to avoid complex and monolithic cooperative connectivity and minimum cost constraint set equations.

The cooperative connectivity models derived when there are $K$ channels available (Fig. 3 ) are the most general set. The cooperative connectivity models derived when there are

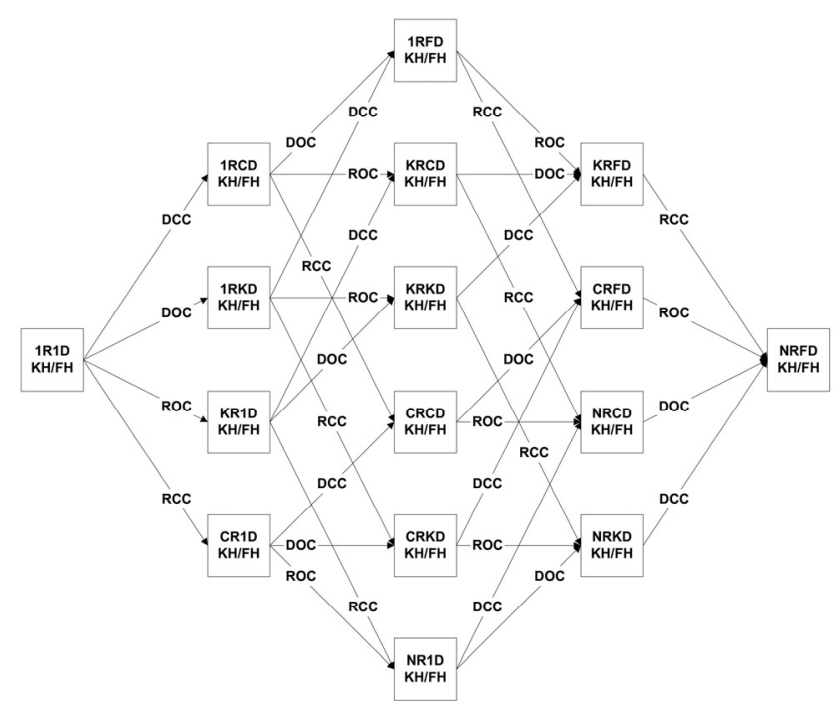

Fig. 3. Cooperative connectivity model transitions for KCA.

2 channels available (Fig. 4) are a subset of the models derived when there are $K$ channels available, with the reduction resulting from additional system resource constraints and intersection between models. The majority of cooperative connectivity models result from constraint combinations with less than $N$ channels available (Fig. 5). Only the models with full relay connectivity, FR1DFH and FRFDFH, are exclusive to constraint combinations with $N$ channels available. This means that full cooperative connectivity is only achievable when $N$ orthogonal channels are available, implying that 


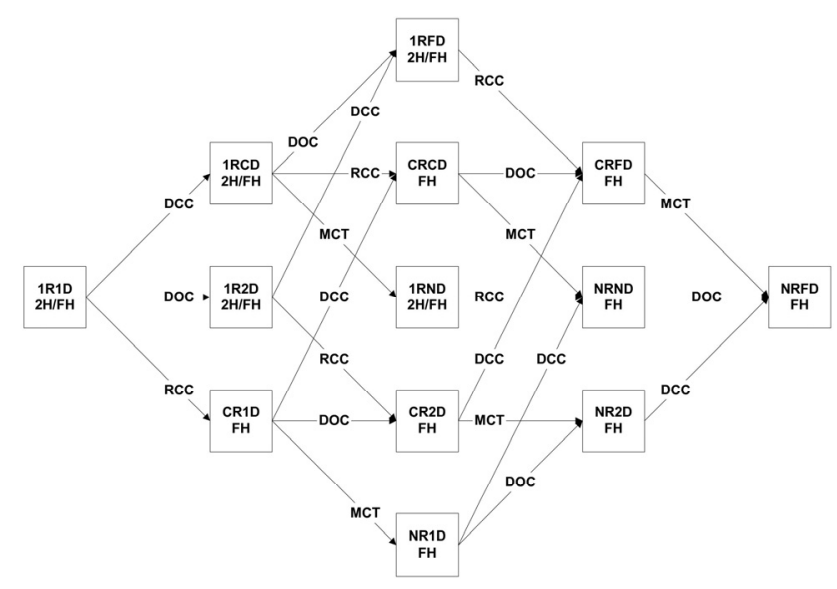

Fig. 4. Cooperative connectivity model transitions for $2 \mathrm{CA}$.

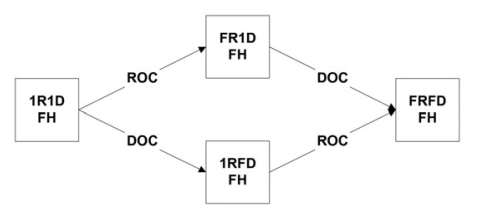

Fig. 5. Cooperative connectivity model transitions for NCA.

the FRFDFH model will be very expensive to implement in practice for even a moderate number of relay terminals.

\section{Minimum Cost Constraint Sets}

The sets of constraints that result in the different cooperative connectivity models while minimizing the system cost (the minimum cost constraint sets) are derived in this section. These minimum cost constraint sets are important in that they indicate the optimal ways to implement the different cooperative connectivity models. The minimum cost constraint sets assume the following ordering, with increasing system cost, of the system resource constraints: DOC, DCC, ROC, IIC, RCC, MCT, KCA, and NCA. It is assumed that constraints involving additional power, interference, or channels within the network itself have a greater system cost than constraints involving more complex terminals. This is based on the underlying assumption that the cost of hardware complexity generally decreases with time while the cost of bandwidth is fixed [47]. It is further assumed that constraints involving more complex relays terminals have a greater system cost than constraints involving more complex destination terminals because in general they affect more terminals in a multihop transmission path. It is important to note that a different ordering of system resource constraints with respect to increasing system cost would result in different minimum cost constraint sets for some of the cooperative connectivity models.

The minimum cost constraint set equations presented in Fig. 2 specify the relationship between the characterizing parameters of the cooperative connectivity models and the presence of each system resource in the minimum cost constraint set. Together with the previously presented cooperative connectivity equations, these equations address the second problem class listed in the introduction: given a desired level of cooperative connectivity, determine the possible (and lowest cost) sets of system resources that can be used to achieve it. Note that when a particular system resource is never present for a given number of channels available it does not imply that the corresponding system resource does not have any impact, only that the same cooperative connectivity can be achieved using an alternative set of system resources with lower system cost.

Figs. 3-5 respectively show the transitions between the different cooperative connectivity models for constraint changes following the minimum cost constraint sets when there are $K, 2$, and $N$ channels available. These transition diagrams show the paths of increasing cooperative connectivity that can be followed without wasting any system resources, which would occur if a particular allocated system resource does not contribute to improving the cooperative connectivity. Increasing cooperative connectivity refers to the fact that as more system resources are available it is possible to add more active links between pairs of terminals. Each transition in the transition diagrams corresponds to the inclusion of an additional system resource and the possibility of adding more active links that rely on that system resource, therefore increasing the achievable cooperative connectivity. The boxes with 'KH/FH' represent two cooperative connectivity models with a different maximum length of the longest multihop path of the network. Transitions between 'KH' and 'FH' cooperative connectivity models correspond to the IIC system resource constraint being lifted. The boxes with only 'FH' indicate that the corresponding ' $\mathrm{KH}$ ' cooperative connectivity model does not exist for the given number of available channels. Transitions are in the direction of decreased system resource constraints.

Transitions that decrease system resource constraints without improving the cooperative connectivity are not shown. For example, adding MCT when the cooperative connectivity model is 1 R1DFH brings no benefit since the terminals are not able to diversity combine the new channels. Transitions that do not follow the minimum cost constraint sets are also not shown. For example, adding DOC when the cooperative connectivity model is $1 \mathrm{RNDFH}$ is not optimal since the resultant 1 RFDFH cooperative connectivity model does not require MCT. Together with the previously presented cooperative connectivity and minimum cost constraint set equations, these transition diagrams address the third and fourth problem classes listed in the introduction.

\section{LITERATURE MAPPING}

This section summarizes the mapping of the cooperative connectivity models to various distributed spatial diversity techniques presented in the literature. The list of presented references is not intended to be comprehensive, but is generally representative of the current state of the literature at the time of writing. Fig. 6 shows this mapping, indicating the name of the distributed spatial diversity technique, the corresponding references, the corresponding cooperative connectivity model of the current framework, and the channel allocation assumed in the references. Those references with a channel allocation of "N/A" present information theoretic results that are independent of channel allocation. This mapping highlights that although the literature published so far has only started to 


\begin{tabular}{|c|c|c|c|}
\hline Distributed Spatial Diversity Technique & References & Model & \# Chnls \\
\hline 2-hop multihop without diversity & [9] & 1R1D2H & 2CA \\
\hline Conventional 2-hop relaying & [28] & 1R1D2H & $2 \mathrm{CA}$ \\
\hline Relayed transmission & {$[26],[72]$} & 1R1D2H & $2 \mathrm{CA}$ \\
\hline Multihop without diversity & {$[9]$} & 1R1DFH & $2 \mathrm{CA}$ \\
\hline Multi-hop cooperation & {$[57]$} & 1R1DFH & NCA \\
\hline Multihop relaying & {$[25],[34],[53]$} & 1R1DFH & NCA \\
\hline Wireless network $\mathrm{O}(\sqrt{ } \mathrm{n})$ & {$[20]$} & 1R1DFH & NCA \\
\hline Multihop relaying & [35] & 1R1DFH & N/A \\
\hline 2-hop multihop diversity & {$[8],[9]$} & 1R2D2H & $2 \mathrm{CA}$ \\
\hline Coded cooperation & {$[30],[31],[32],[51]$} & 1R2D2H & $2 \mathrm{CA}$ \\
\hline Cooperative coding & {$[45],[64]$} & 1R2D2H & $2 \mathrm{CA}$ \\
\hline Cooperative diversity & {$[27],[39],[40],[42],[43]$} & 1R2D2H & $2 \mathrm{CA}$ \\
\hline Cooperative protocols I, II, and III & {$[50]$} & 1R2D2H & $2 \mathrm{CA}$ \\
\hline Cooperative space-time delay coding & [52] & 1R2D2H & $2 \mathrm{CA}$ \\
\hline Cooperative superposition modulation & [44] & 1R2D2H & $2 \mathrm{CA}$ \\
\hline Distributed turbo coded diversity & [76] & 1R2D2H & $2 \mathrm{CA}$ \\
\hline Relayed block markov transmission & {$[13]$} & 1R2D2H & $2 \mathrm{CA}$ \\
\hline MIMO relay channel & [67] & 1R2D2H & N/A \\
\hline Relay channel & [14] & 1R2D2H & N/A \\
\hline Multihop, multi-branch cooperation & [57] & 1RKDKH & NCA \\
\hline Non-interfering multi-path transmission & {$[55]$} & 1RKDKH & NCA \\
\hline 2-hop MSE relaying & {$[36]$} & 1RCD2H & $2 \mathrm{CA}$ \\
\hline Coherent cooperative transmission & [66] & 1RCD2H & $2 \mathrm{CA}$ \\
\hline Interference relay network & [48] & 1RCD2H & $2 \mathrm{CA}$ \\
\hline Linear relaying & [70] & 1RCD2H & 2CA \\
\hline Parallel relays w space-time modulations & [29] & 1RCD2H & $2 \mathrm{CA}$ \\
\hline User cooperation & {$[7],[37],[61]$} & 1RCD2H & $2 \mathrm{CA}$ \\
\hline Virtual antenna array & [5] & 1RCD2H & $2 \mathrm{CA}$ \\
\hline Parallel relay network & {$[60]$} & 1RCD2H & N/A \\
\hline Interfering multi-path transmission & [55] & 1RCDKH & KCA \\
\hline 2-hop cooperative relaying & {$[23],[24]$} & 1RFD2H & $2 \mathrm{CA}$ \\
\hline 2-hop relay network & {$[69]$} & 1RFD2H & $2 \mathrm{CA}$ \\
\hline Collaborative coding & [46] & 1RFD2H & $2 \mathrm{CA}$ \\
\hline Distributed Alamouti system & [3] & 1RFD2H & $2 \mathrm{CA}$ \\
\hline Distributed space-time coding & [33],[41],[49] & 1RFD2H & $2 \mathrm{CA}$ \\
\hline Dynamic decoded and forward & {$[4]$} & 1RFD2H & $2 \mathrm{CA}$ \\
\hline Network path selection diversity & [6] & 1RFD2H & $2 \mathrm{CA}$ \\
\hline Non-orthogonal amplify and forward & [4] & 1RFD2H & 2CA \\
\hline Relay assisted MIMO channel & [56] & 1RFD2H & $2 \mathrm{CA}$ \\
\hline Single-stage cooperative relaying & {$[53]$} & 1RFD2H & $2 \mathrm{CA}$ \\
\hline Cooperative network & {$[2]$} & 1RFD2H & NCA \\
\hline Multi-branch cooperation & [57] & 1RFD2H & NCA \\
\hline Multi-user spatial diversity & [17] & 1RFD2H & NCA \\
\hline Repetition-based cooperative diversity & {$[41]$} & 1RFD2H & NCA \\
\hline $\mathrm{C}(\mathrm{m})$ cooperative diversity where $\mathrm{K}=\mathrm{m}+1$ & [58] & KRFDFH & NCA \\
\hline Cascaded (K-1)-hop cooperative diversity & {$[28]$} & KRKDFH & KCA \\
\hline Two-level leapfrog scheme with $\mathrm{K}=2$ & [22] & KRKDFH & KCA \\
\hline Amplify-and-forward MIMO tunnel & [55] & CRKDKH & KCA \\
\hline Distributed MIMO multihop system & {$[15]$} & CRCDKH & KCA \\
\hline Multi-stage cooperative relaying & [53] & CRCDKH & KCA \\
\hline Opportunistic large array & [59], [63] & CRCDKH & KCA \\
\hline $\mathrm{C}(\mathrm{N}-1)$ cooperative diversity where $\mathrm{K}=\mathrm{N}$ & [58] & FRFDFH & NCA \\
\hline Full cooperative relaying & [28] & FRFDFH & NCA \\
\hline Multihop diversity & {$[8],[9]$} & FRFDFH & NCA \\
\hline Relay network & {$[18]$} & FRFDFH & NCA \\
\hline Cooperative wireless system & {$[73],[74]$} & FRFDFH & N/A \\
\hline Decode / compress and forward & {$[38]$} & FRFDFH & N/A \\
\hline Multiple level relay channel & [71] & FRFDFH & N/A \\
\hline Wireless network $O(n)$ & [21] & FRFDFH & N/A \\
\hline
\end{tabular}

Fig. 6. Mapping of cooperative connectivity models to the literature.

explore the many possible cooperative connectivity models there is already a broad range of connectivity and channel allocation assumptions. It also illustrates the wide variation in terminology used in the literature. The developed framework allows us to compare the cooperative connectivity and system resource requirements of the proposed distributed spatial diversity techniques and analyze the efficiency, in comparison to the minimum cost constraint sets, of the available system resources assumed in the literature. Due to space constraints, we do not summarize herein the sets of available system resources actually assumed in the included references.

\section{CONCLUSION}

This paper develops a framework for modeling the ways that cooperating terminals can be connected to each other in wireless relay networks and the relationship between constraints on the available system resources and the achievable cooperative connectivity. Cooperative connectivity equations are derived that specify the relationship between system resource con- straints and achievable cooperative connectivity. The resulting cooperative connectivity models defined by the achievable combinations of communication links between cooperating wireless terminals are associated with their minimum cost constraint sets and mapped to relevant diversity techniques presented in the literature. It is indicated that the literature published so far has only started to explore the many possible cooperative connectivity models and the richness of the problem domain. Finally, it is straightforward to see how this framework can provide value as an analysis tool, for example by leveraging it in a series of probability of outage or error simulations that compare the different cooperative connectivity models in order to isolate the performance impact of specific system resource constraints.

\section{REFERENCES}

[1] S. Alamouti, "A simple transmit diversity technique for wireless communications," IEEE J. Select. Areas Commun., vol. 16, no. 8, pp. 1451-1458, Oct. 1998.

[2] P. Anghel and M. Kaveh, "Exact symbol error probability of a cooperative network in a Rayleigh fading environment," IEEE Trans. Wireless Commun., vol. 3, no. 5, pp. 1416-1421, Sep. 2004.

[3] P. Anghel, G. Leus, and M. Kaveh, "Multi-user space-time coding in cooperative networks," in Proc. IEEE International Conf. Acoustics, Speech, Signal Processing, Apr. 2003, vol. 4, pp. 73-76.

[4] K. Azarian, H. Gamal, and P. Schniter, "On the achievable diversitymultiplexing tradeoff in half-duplex cooperative channels," in Proc. Allerton Conf. Commun., Control, Computing, Sep. 2004, pp. 41524172.

[5] S. Barbarossa and G. Scutari, "Cooperative diversity through virtual arrays in multihop networks," in Proc. IEEE International Conf. Acoustics, Speech, Signal Processing, Apr. 2003, vol. 4, pp. 209-212.

[6] A. Bletsas, A. Khisti, D. P. Reed, and A. Lippman, "A simple cooperative diversity method based on network path selection," IEEE J. Select. Areas Commun., vol. 24, no. 3, pp. 659-672, Mar. 2006.

[7] H. Boelcskei, R. U. Nabar, O. Oyman, and A. J. Paulraj, "Capacity scaling laws in MIMO relay networks," IEEE Trans. Wireless Commun., vol. 5, no. 6, pp. 1433-1444, June 2006.

[8] J. Boyer, D. D. Falconer, and H. Yanikomeroglu, "A theoretical characterization of multihop wireless communications channels with diversity," in Proc. IEEE GLOBECOM, Nov. 2001, pp. 841-845.

[9] J. Boyer, D. D. Falconer, and H. Yanikomeroglu, "Multihop diversity in wireless relaying channels," IEEE Trans. Commun., vol. 52, no. 10, pp. 1820-1830, Oct. 2004.

[10] J. Boyer, D. D. Falconer, and H. Yanikomeroglu, "On the aggregate SNR of amplified relaying channels," in Proc. IEEE GLOBECOM, Nov.-Dec. 2004, vol. 5, pp. 3394-3398.

[11] J. Boyer, D. D. Falconer, and H. Yanikomeroglu, "On the impact of system resource constraints on wireless relaying channels," in Proc. IEEE International Conf. Commun., May 2005, vol. 5, pp. 3266-3270.

[12] J. Boyer, D. Falconer, and H. Yanikomeroglu, "On the maximum diversity order of wireless relay networks: Common codebook generation," IEEE Trans. Commun., submitted, 2005.

[13] X. Cai, Y. Yao, and G. Giannakis, "Achievable rates in low-power relay links over fading channels," IEEE Trans. Commun., vol. 53, no. 1, pp. 184-194, Jan. 2005.

[14] T. Cover and A. Gamal, "Capacity theorems for the relay channel," IEEE Trans. Inform. Theory, vol. 25, no. 5, pp. 572-584, Sep. 1979.

[15] M. Dohler, A. Gkelias, and H. Aghvami, "A resource allocation strategy for distributed MIMO multi-hop communication systems," IEEE Commun. Lett., vol. 8, no. 2, pp. 99-101, Feb. 2004.

[16] X. Dong and N. Beaulieu, "Optimal maximal ratio combining with correlated diversity branches," IEEE Commun. Lett., vol. 6, no. 1, pp. 22-24, Jan. 2002.

[17] V. Emamian, P. Anghel, and M. Kaveh, "Multi-user spatial diversity in a shadow-fading environment," in Proc. IEEE Veh. Technol. Conf., Sep. 2002, vol. 1, pp. 573-576.

[18] M. Gastpar and M. Vetterli, "On the capacity of large Gaussian relay networks," IEEE Trans. Inform. Theory, vol. 51, no. 3, pp. 765-779, Mar. 2005.

[19] D. Gore, S. Sandhu, and A. Paulraj, "Delay diversity codes for frequency selective channels," in Proc. IEEE International Conf. Commun., Apr--May 2002, vol. 3, pp. 1949-1953. 
[20] P. Gupta and P. Kumar, "The capacity of wireless networks," IEEE Trans. Inform. Theory, vol. 46, no. 2, pp. 388-404, Mar. 2000.

[21] P. Gupta and P. Kumar, "Towards an information theory of large networks: An achievable rate region," IEEE Trans. Inform. Theory, vol. 49, no. 8, pp. 1877-1894, Aug. 2003.

[22] M. Haenggi, "Analysis and design of diversity schemes for ad hoc wireless networks," IEEE J. Select. Areas Commun., vol. 23, no. 1, pp. 19-27, Jan. 2005.

[23] I. Hammerstrom, M. Kuhn, and A. Wittneben, "Cooperative diversity by relay phase rotations in block fading environments," in Proc. IEEE Workshop Signal Processing Advances Wireless Commun., July 2004, pp. 293-297.

[24] I. Hammerstrom, M. Kuhn, and A. Wittneben, "Impact of relay gain allocation on the performance of cooperative diversity networks," in Proc. IEEE Veh. Technol. Conf., Sep. 2004, vol. 3, pp. 1815-1819.

[25] M. Hasna and M. Alouini, "End-to-end performance of transmission systems with relays over Rayleigh fading channels," IEEE Trans. Wireless Commun., vol. 2, no. 6, pp. 1126-1131, Nov. 2003.

[26] M. Hasna and M. Alouini, "Harmonic mean and end-to-end performance of transmission systems with relays," IEEE Trans. Commun., vol. 52, no. 1, pp. 130-135, Jan. 2004.

[27] P. Herhold, E. Zimmermann, and G. Fettweis, "A simple cooperative extension to wireless relaying," in Proc. International Zurich Seminar Commun., Feb. 2004, pp. 36-39.

[28] P. Herhold, E. Zimmerman, and G. Fettweis, "Co-operative multi-hop transmission in wireless networks," Computer Networks J., vol. 49, no. 3, pp. 299-324, Oct. 2005.

[29] Y. Hua, Y. Mei, and Y. Chang, "Parallel wireless mobile relays with space-time modulations," in Proc. IEEE Workshop Statistical Signal Processing, Sep. 2003, pp. 375-378.

[30] T. Hunter and A. Nosratinia, "Cooperation diversity through coding," in Proc. IEEE International Symposium Inform. Theory, June/July 2002, p. 220.

[31] T. Hunter, S. Sanayei, and A. Nosratinia, "Outage analysis of coded cooperation," IEEE Trans. Inform. Theory, vol. 52, no. 2, pp. 375-391, Feb. 2006.

[32] M. Janani, A. Hedayat, T. Hunter, and A. Nosratinia, "Coded cooperation in wireless communications: Space-time transmission and iterative decoding," IEEE Trans. Signal Processing, vol. 52, no. 2, pp. 362-371, Feb. 2004.

[33] Y. Jing and B. Hassibi, "Cooperative diversity in wireless relay networks with multiple-antenna nodes," in Proc. International Symposium Inform. Theory, Sep. 2005, pp. 815-819.

[34] G. Karagiannidis, D. A. Zoyas, N. C. Sagias, T. A. Tsiftsis, and P. T Mathiopoulos, "Multihop communications with fixed-gain relays over generalized fading channels," in Proc. IEEE GLOBECOM, Nov.-Dec. 2004, pp. 36-40.

[35] G. Karagiannidis, T. Tsiftsis, and R. Mallik, "Bounds for multihop relaying communications in Nakagami- $m$ fading," IEEE Trans. Commun., vol. 54, no. 1, pp. 18-22, Jan. 2006.

[36] N. Khajehnouri and A. Sayed, "Multi-relay strategy for imperfect channel information in sensors networks," in Proc. European Signal Processing Conf., Sep. 2005.

[37] M. Khojastepour, A. Sabharwal, and B. Aazhang, "Improved achievable rates for user cooperation and relay channels," in Proc. IEEE International Symposium Inform. Theory, June 2004, p. 4.

[38] G. Kramer, M. Gastpar, and P. Gupta, "Cooperative strategies and capacity theorems for relay networks," IEEE Trans. Inform. Theory, vol. 51, no. 9, pp. 3037-3063, Sep. 2005.

[39] J. Laneman and G. Wornell, "Energy-efficient antenna sharing and relaying for wireless networks," in Proc. IEEE Wireless Commun. Networking Conf., Sep. 2000, pp. 7-12.

[40] J. Laneman and G. Wornell, "Exploiting distributed spatial diversity in wireless networks," in Proc. Allerton Conf. Commun., Control, Computing, Oct. 2000.

[41] J. Laneman and G. Wornell, "Distributed space-time-coded protocols for exploiting cooperative diversity in wireless networks," IEEE Trans. Inform. Theory, vol. 49, no. 10, pp. 2415-2425, Oct. 2003.

[42] J. Laneman, D. Tse, and G. Wornell, "Cooperative diversity in wireless networks: Efficient protocols and outage behavior," IEEE Trans. Inform. Theory, vol. 50, no. 12, pp. 3062-3080, Dec. 2004.

[43] J. Laneman, "Network coding gain of cooperative diversity," in Proc. IEEE MILCOM, Oct.-Nov. 2004, vol. 1, pp. 106-112.

[44] E. Larsson and B. Vojcic, "Cooperative transmit diversity based on superposition modulation," IEEE Commun. Lett., vol. 9, no. 9, pp. 778-780, Sep. 2005.
[45] Z. Lin, E. Erkip, and A. Stefanov, "Cooperative regions for coded cooperative systems," in Proc. IEEE GLOBECOM, Nov.-Dec. 2004, vol. 1, pp. 21-25.

[46] P. Mitran, H. Ochiai, and V. Tarokh, "Space-time diversity enhancements using collaborative communications," IEEE Trans. Inform. The ory, vol. 51, no. 6, pp. 2041-2057, June 2005.

[47] G. Moore, "Cramming more components onto integrated circuits," Electronics Magazine, vol. 38, no. 8, pp. 114-117, Apr. 1965.

[48] V. Morgenshtern, H. Boelcskei, and R. Nabar, "Distributed orthogonalization in large interference relay networks," in Proc. International Symposium Inform. Theory, Sep. 2005, pp. 1211-1215.

[49] R. Mudumbai, G. Barriac, and U. Madhow, "Spread-spectrum techniques for distributed space-time communication in sensor networks," in Proc. Asilomar Conf. Signals, Syst., Computers, Nov. 2004, pp. 908912.

[50] R. Nabar, H. Boelcskei, and F. Kneubuhler, "Fading relay channels: Performance limits and space-time signal design," IEEE J. Select. Areas Commun., vol. 22, no. 6, pp. 1099-1109, Aug. 2004.

[51] A. Nosratinia, T. Hunter, and A. Hedayat, "Cooperative communication in wireless networks," IEEE Commun. Mag., vol. 42, no. 10, pp. 74-80, Oct. 2004.

[52] T. Oechtering and A. Sezgin, "A new cooperative transmission scheme using the space-time delay code," ITG Workshop Smart Antennas, Mar. 2004, pp. 41-48.

[53] R. Pabst, B. H. Walke, D. C. Schultz, P. Herhold, H. Yanikomeroglu, S. Mukherjee, H. Viswanathan, M. Lott, W. Zirwas, M. Dohler, H. Aghvami, D. D. Falconer, and G. P. Fettweis, "Relay-based deployment concepts for wireless and mobile broadband radio," IEEE Commun. Mag., vol. 42, no. 9, pp. 80-89, Sep. 2004.

[54] J. Proakis, Digital Communications, 4th ed. New York: McGraw-Hill, Inc., 2001.

[55] B. Rankov and A. Wittneben, "Distributed spatial multiplexing in a wireless network, in Proc. Asilomar Conf. Signals, Syst., Computers, Nov. 2004, pp. 1932-1937.

[56] B. Rankov and A. Wittneben, "On the capacity of relay-assisted wireless MIMO channels," in Proc. IEEE Workshop Signal Processing Advances Wireless Commun., July 2004, pp. 323-327.

[57] A. Ribeiro, X. Cai, and G. Giannakis, "Symbol error probabilities for general cooperative links," IEEE Trans. Wireless Commun., vol. 4, no. 3, pp. 1264-1273, May 2005.

[58] A. Sadek, W. Su, and K. Liu, "A class of cooperative communication protocols for multi-node wireless networks," in Proc. IEEE International Workshop Signal Processing Advances Wireless Commun., June 2005, pp. 560-564.

[59] A. Scaglione and Y. Hong, "Opportunistic large arrays: Cooperative transmission in wireless multihop ad hoc networks to reach far distances," IEEE Trans. Signal Processing, vol. 51, no. 8, pp. 2082-2092, Aug. 2003.

[60] B. Schein and R. Gallager, "The Gaussian parallel relay network," in Proc. IEEE International Symposium Inform. Theory, June 2000, p. 22.

[61] A. Sendonaris, E. Erkip, and B. Aazhang, "User cooperation diversity - Part I: System description / Part II: Implementation aspects and performance analysis," IEEE Trans. Commun., vol. 51, no. 11, pp. 1927-1948, Nov. 2003.

[62] N. Seshadri and J. Winters, "Two signaling schemes for improving the error performance of frequency-division-duplex (FDD) transmission systems using transmitter antenna diversity," in Proc. IEEE VTC, May 1993, pp. 508-511.

[63] B. Sirkeci-Mergen and A. Scaglione, "Signal acquisition for cooperative transmissions in multi-hop ad-hoc networks," in Proc. IEEE ICASSP, May 2004, pp. 345-348.

[64] A. Stefanov and E. Erkip, "Cooperative coding for wireless networks," IEEE Trans. Commun., vol. 52, no. 9, pp. 1470-1476, Sep. 2004.

[65] D. Tse, P. Viswanath, and L. Zheng, "Diversity-multiplexing tradeoff in multiple-access channels," IEEE Trans. Inform. Theory, vol. 50, no. 9, pp. 1859-1874, Sep. 2004.

[66] Y. Tu and G. Pottie, "Coherent cooperative transmission from multiple adjacent antennas to a distant stationary antenna through AWGN channels," in Proc. IEEE Veh. Technol. Conf., May 2002, vol. 1, pp. $130-134$.

[67] B. Wang, J. Zhang, and A. Madsen, "On the capacity of MIMO relay channels," IEEE Trans. Inform. Theory, vol. 51, no. 1, pp. 29-43, Jan. 2005.

[68] A. Wittneben, "A new bandwidth efficient transmit antenna modulation diversity scheme for linear digital modulation," in Proc. IEEE International Conf. Commun., May 1993, vol. 3, pp. 1630-1634. 
[69] A. Wittneben and B. Rankov, "Impact of cooperative relays on the capacity of rank-deficient MIMO channels," in Proc. IST Summit Mobile Wireless Commun., June 2003, pp. 421-425.

[70] A. Wittneben and B Rankov, "Distributed antenna systems and linear relaying for gigabit MIMO wireless," in Proc. IEEE Veh. Technol. Conf., Sep. 2004, vol. 3, pp. 3624-3630.

[71] L. Xie and P. Kumar, "An achievable rate for the multiple-level relay channel," IEEE Trans. Inform. Theory, vol. 51, no. 4, pp. 1348-1358, Apr. 2005.

[72] L. Yang, H. Hasna, and M. Alouini, "Average outage duration of multihop communication systems with regenerative relays," IEEE Trans. Wireless Commun., vol. 4, no. 4, pp. 1366-1371, July 2005.

[73] M. Yuksel and E. Erkip, "Diversity in relaying protocols with amplify and forward," in Proc. IEEE GLOBECOM, Dec. 2003, vol. 4, pp. 2025-2029.

[74] M. Yuksel and E. Erkip, "Diversity-multiplexing tradeoff in cooperative wireless systems," in Proc. Conf. Inform. Sciences Syst., 2006.

[75] Q. Zhang, "Maximal-ratio combining over Nakagami fading channels with an arbitrary branch covariance matrix," IEEE Trans. Veh. Technol., vol. 48, no. 4, pp. 1141-1150, July 1999.

[76] B. Zhao and M. Valenti, "Distributed turbo coded diversity for relay channel," IEE Electron. Lett., vol. 39, no. 10, pp. 786-787, 2003.

[77] L. Zheng and D. Tse, "Diversity and multiplexing: A fundamental tradeoff in multiple-antenna channels," IEEE Trans. Inform. Theory, vol. 49, no. 5, pp. 1073-1096, May 2003. 